\title{
Heterogeneous Target Assignment to Robotic Fiber Positioner Systems
}

\author{
Matin Macktoobian ${ }^{1}$, Denis Gillet ${ }^{1}$, and Jean-Paul Kneib ${ }^{2}$
}

\begin{abstract}
The target assignment methods applied to robotic fiber positioners are only applicable to homogeneous sets of targets to be observed. However, different batches of robotic fiber positioners available at the focal plane of a telescope can be simultaneously used in various applications. Those applications may be intrinsically different, say, some may seek astronomical observations, whereas the others may focus on space operations such as space debris detection. The current target assignment algorithms cannot handle these heterogeneous scenarios. This paper proposes an efficient multilinear algorithm to assign robotic fiber positioners to heterogeneous targets. We classify the targets based on their required exposure times. We also take the priority of observations compared to other operations into account during an assignment processes. Our algorithm assigns a bundle of robotic positioners to each dynamic target to efficiently detect it. We finally illustrate our algorithm's application using a simulated example.
\end{abstract}

\section{INTRODUCTION}

Observational astronomy has recently sought the generation of the map of the observable universe. This class of maps paves the way for the evolution analysis of the universe and the distribution of dark matter all over it. Electromagnetic waves, such as visible light, infrared, and gamma ray, radiated from astronomical objects can be received by fibers, and those signals are processed by spectrographs to generate spectrographic surveys of the sky [1]. Many projects, including DESI [2], [3], MOONS [4], PFS [5], SLOAN [6], [7], etc., have been defined to develop telescopes with spectroscopic capabilities. Each fiber is attached to a two-degreeof-freedom rotational-rotational robotic positioner [8], so it can rotate in a circular workspace to point to some particular desired directions. The overall set of positioners is mounted at the focal plane area of a telescope. The signals received by the robotic positioners are transferred to a spectrograph which generates the desired map.

Each observation often encompasses many objects to be observed. Thus, some algorithms were proposed to assign each target to a specific fiber. Random assignment [9] is the simplest approach by which no specific optimization criteria can be taken into account. To obtain more efficient assignments, the drainage algorithm [10] was proposed whose assignment gain is almost five per cent higher than

\footnotetext{
${ }^{1}$ M. Macktoobian and D. Gillet are with the School of Engineering, Swiss Federal Institute of Technology in Lausanne (EPFL), 1015 Lausanne, Switzerland \{matin.macktoobian, denis.gillet\} depfl.ch

${ }^{2} \mathrm{~J}$-P. Kneib is with the School of Basic Sciences, Swiss Federal Institute of Technology in Lausanne (EPFL), 1015 Lausanne, Switzerland jean-paul.kneib@epfl.ch

This work was financially supported by the Swiss National Science Foundation (SNF) grant number 20FL21_185771 and the SLOAN ARC/EPFL agreement number SSP523.
}

that of the random target assignment. This method uses a tiling process based on which the applied assignments to fibers are optimized. Namely, the method moves the targets between different lists of fibers each of which can observe a specific set of targets. Then, a target is assigned to a list with the shortest number of observable targets. In other words, this method aims to homogenize the target-to-positioner ratio corresponding to a fiber-target system. However, this strategy does not assume any physical size for positioners to neglect the potential collisions between robotic positioners, which is not a realistic assumption. Target-based assignment [11], [12] is another method which assigns positioners to targets instead of targets to positioners. The target assignment efficiency of this algorithm surpasses that of the drainage algorithm at the cost of increasing the computational complexity of the assignment process.

The cited assignment approaches are only applicable to the set of homogeneous targets. In particular, when all of the targets (for example, astronomical objects) and their desired operations (for example, astronomical observations) are the same for all positioners, then one can use the quoted algorithms to resolve assignment processes. However, if various subsets of positioners are supposed to be used to handle different types of operations, then the algorithms above shall not be applicable. For example, active space debris mitigation [13] seeks the strategies to detect space debris at long ranges. Since space debris radiate near-infrared waves, infrared fibers [14] can be used to detect them at the range of low earth orbit (LEO), which is an interesting region in view of debris mitigation. As another motivation, the number of available fibers may exceed the number of the desired targets to be observed. Thus, one would like to assign some of the free fibers to another application such as space debris detection, as explained above. In this case, the assignment procedure is heterogeneous, in that there are two classes of targets which are substantially different and need to be treated differently in view of the fiber assignment procedure. Heterogeneous assignment maximizes the operational capacity of the fibers of a telescope such that the fibers which are not assigned to any observational target may be assigned to other targets corresponding to a secondary application.

This paper proposes a multilinear-time algorithm to assign robotic fiber positioners of a telescope to more than one operational application. In view of our heterogeneous algorithm, the targets are classified into two categories: the observational targets which are often very far from the earth and move very slow, so one can consider them static; the second group include the targets which are relatively closer to the earth and move faster, so one takes them as dynamic 


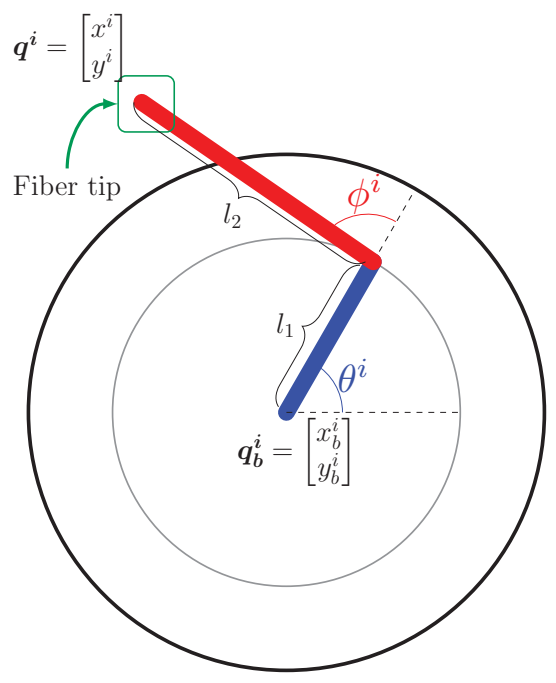

Fig. 1. The schematic of a typical robotic positioner [6]

targets into account. Since astronomical observations are the main applications of fiber positioners, we divide observation targets to two groups of high-priority and low-priority targets. The high-priority targets are assigned to fibers ahead of the dynamic targets. Finally, we assign the low-priority targets to any remaining fiber which has not yet been assigned to either a high-priority static or dynamic target. As we explain in detail further, our heterogeneous algorithm assigns the minimum number of positioners, called bundle, to a dynamic target which is necessary to detect it. Thus, our strategy is optimized in view of bundle formation. In other words, minimizing the bundle size provides more unallocated fibers to be assigned to static targets. Since our algorithm takes two partitions of low- and high-priority static targets into account, it is flexible enough to manage the assignment completeness. To be specific, if the fiber assignment to dynamic targets is not satisfactorily complete, then one may decrease the number of high-priority static targets to provide more available fibers to dynamic targets. Overall, there is generally a trade-off between the complete assignment of fibers to static targets and those of to dynamic ones.

The remainder of this paper is organized as follows. Section II briefly reviews the general characteristics of fibers and robotic positioners assigned to them. Heterogeneous target assignment process is investigated in Section III. Namely, Section III-A describes a bundle formation method to select a minimal subset of available fiber positioners to each dynamic target. We use the bundle formation method to propose our heterogeneous target assignment algorithm in Section III-B. We also show that the algorithm is multilinear in view of computational complexity. Section IV solves a heterogeneous target assignment scenario using our algorithm to illustrate its efficiency.

\section{Robotic Fiber Positioners}

We briefly introduce fibers and robotic fiber positioners used in cosmic spectroscopy. A fiber is a string medium

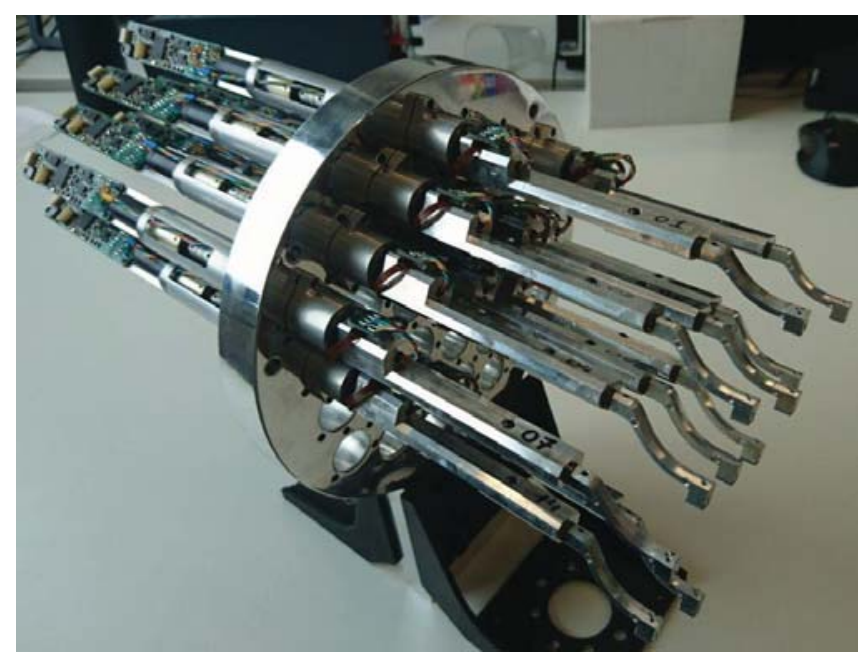

Fig. 2. A set of robotic positioners mounted on a typical focal plane [6]

at the tip of which a sensor detects a specific type of electromagnetic waves radiated by a particular object in the sky. Then, the signal is transmitted to a spectrograph, which processes it to add the trace of the observed object to the map it generates. Based on the typical application of fibers in cosmological observation, a swarm of fibers yields massive spectroscopic surveys.

A set of robotic positioners are mounted on a particular area of its hosting telescope called focal plane. Each robotic positioner is a planar rotational-rotational manipulator whose end-effector, called ferrule, shall reach the point at which its fiber has to observe an object based on a particular observation or application. The forward kinematics corresponding to the workspace of each positioner is described as below.

$$
\boldsymbol{q}^{i}=\boldsymbol{q}_{\boldsymbol{b}}^{i}+\left[\begin{array}{cc}
\cos \left(\theta^{i}\right) & \cos \left(\theta^{i}+\phi^{i}\right) \\
\sin \left(\theta^{i}\right) & \sin \left(\theta^{i}+\phi^{i}\right)
\end{array}\right] \boldsymbol{l}
$$

Here the $i^{\text {th }}$ positioner is located at $\boldsymbol{q}^{i}=\left[\begin{array}{ll}x^{i} & y^{i}\end{array}\right]^{\top}$ with respect to a universal frame attached to the focal plane of the host telescope. $\boldsymbol{q}_{\boldsymbol{b}}^{\boldsymbol{i}}=\left[\begin{array}{ll}x_{b}^{i} & y_{b}^{i}\end{array}\right]^{\top}$ is also the base coordination of the positioner. The lengths of rotational links are represented by $\boldsymbol{l}=\left[\begin{array}{ll}l_{1} & l_{2}\end{array}\right]^{\top}$. The angular positions of the $i^{\text {th }}$ positioner are denoted by $\theta^{i}$ and $\phi^{i}$. The quoted parameters are depicted in Figure 1. Figure 2 illustrates an arrangement of positioners placement in the focal plane of a typical telescope.

The axes of both rotational arms of a positioner are tilted with respect to the axis of its first rotational arm according to some particular specifications. The ferrule is also deviated with respect to the cited axis. So, when the positioner rotates its ferrule, the swept area is vaster than the area corresponding to the base of the positioner. This property gives rise to the expansion of the field of view correspondning to a positioner. In Section III-A, we use this feature as a criterion to select a bundle of positinoers to detect some particular objects. 


\section{Heterogeneous TARget Assignment}

We start from a set of definitions based on which the problem statement of the heterogeneous target assignment is defined. We will also later use these definitions to develop our heterogeneous target assignment algorithm. We already noted that static targets refer to those whose movements are negligible compared to the observation exposure times such as galaxies. Thus, the following definition addresses the notion of "static target" based on the concept of exposure time.

Definition 1 [Static Target]. A target $t_{s}$ is a static target if its movements are negligible during the exposure time required to observe it. Then, the set of static targets is represented by

$$
T_{S}:=\left\{t_{S}^{i} \mid i \in \mathcal{I}\right\}
$$

where $\mathcal{I}$ is an index set.

We partition the set of static targets to two disjoint sets based on their priorities. In other words, the highpriority subset $T_{S}^{H} \subseteq T_{S}$ includes those static targets whose observations are necessary during a particular observation period. Put differently, a robotic positioner has to be assigned to each of those targets. In contrast, the subset $T_{S}^{L} \subseteq T_{S}$ denotes the targets whose observations may be postponed to another observation period if there is currently no free robotic positioner to be assigned to them. Thus, we have $T_{S}:=T_{S}^{H} \dot{U} T_{S}^{L}$.

We also exploit the concept of exposure time to define the notion of "dynamic target" as follows.

Definition 2 [Dynamic Target]. A target $t_{d}$ is a dynamic target if its movements are not negligible during the exposure time required to observe it. Then, the set of static targets is represented by

$$
T_{D}:=\left\{t_{D}^{j} \mid j \in \mathcal{J}\right\},
$$

where $\mathcal{J}$ is an index set.

Then, the overall set of targets is represented by $T:=$ $T_{S} \dot{\cup} T_{D}$.

Definition 3 [Free Positioner]. Let $\mathcal{P}$ be a set of positioners. Then, a positioner $\pi \in \mathcal{P}$ is free if it is not assigned to any specific target.

In general, a single positioner cannot efficiently detect a dynamic target since the target often moves too fast, and the limited workspace of a single positioner is not enough for this purpose. Thus to detect dynamic targets, we construct bundles of positioners which can collectively cover a desired area in which a dynamic target moves.

Definition 4 [Bundle]. Let $\mathcal{P}$ be a set of positioners. Then, $\mathcal{B} \subseteq \mathcal{P}$ is a bundle if its positioners shape a single positioners island.

Remark 1. The definition of free positioner can be extended to define a free bundle which is a bundle whose all positioners are free.

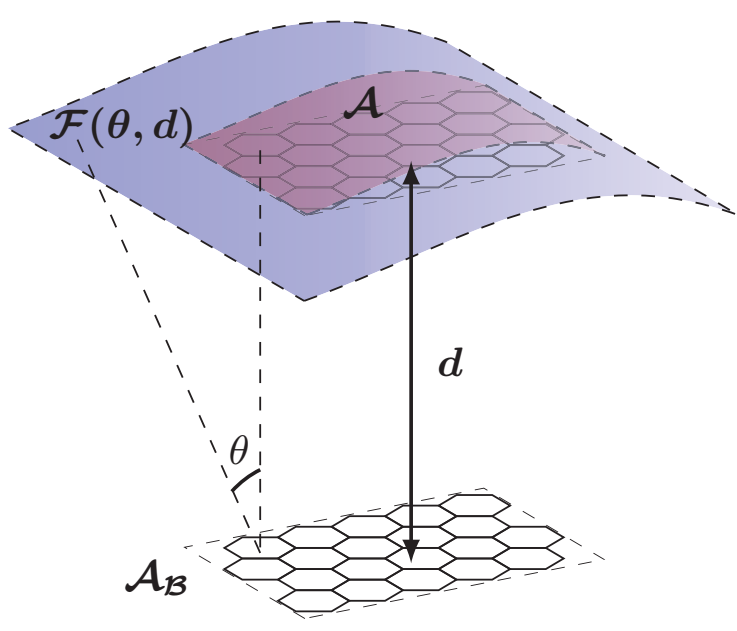

Fig. 3. A typical bundle whose corresponding surface covers a specific area around a particular dynamic object

Thus, we define the heterogeneous target assignment problem as follows.

Problem. Denote by $\mathcal{P}$ a set of robotic positioners, and let $T_{S}$ be a set of static targets subject to some priority specifications. Let also $T_{D}$ be a set of dynamic targets with some region specifications. Assign a

(i) free $\pi \in \mathcal{P}$ to each $t \in T_{S}$;

(ii) free bundle $\mathcal{B} \subseteq \mathcal{P}$ to each $t \in T_{D}$.

In the following two sections, we solve the problem above.

\section{A. Bundle Formation}

Suppose a bundle $\mathcal{B}$ whose base area is $\mathcal{A}_{\mathcal{B}}$ as depicted in Figure 3. Let also $d$ denote the distance of the bundle to a particular dynamic target. We are interested in the area around the target at the distance of $d$ which is observable by $\mathcal{B}$. In other words, the field of view of $\mathcal{B}$ at distance $d$ is of interest. We already cited the tilted axes and the deviated ferrules of positioners. Thus, the field of view of a bundle at distance $d$ encompasses a surface whose area $\mathcal{A}$ is greater than $\mathcal{A}_{\mathcal{B}}$. Denoting the overall deviation by $\theta$, the difference between $\mathcal{A}$ and $\mathcal{A}_{\mathcal{B}}$ depends on both $\theta$ and $d$ as follows

$$
\mathcal{A}=\mathcal{A}_{\mathcal{B}}+\mathcal{F}(\theta, d),
$$

where the definition of the binary function $\mathcal{F}(\cdot, \cdot)$ depends on the geometrical shape of the bundle. Each pair of the arguments associated with $\mathcal{F}(\cdot, \cdot)$ in fact corresponds to a particular dynamic target.

To form a bundle corresponding to a specific dynamic target, the area of the surface, i.e., $\mathcal{A}$, is known because the area in which we are interested in detecting the target is a part of the solutions requirements. Put differently, $\mathcal{A}$ is taken as a region specification into account associated with a specific dynamic target. Thus, one selects some unassigned positioners to shape a bundle with the minimum number of 


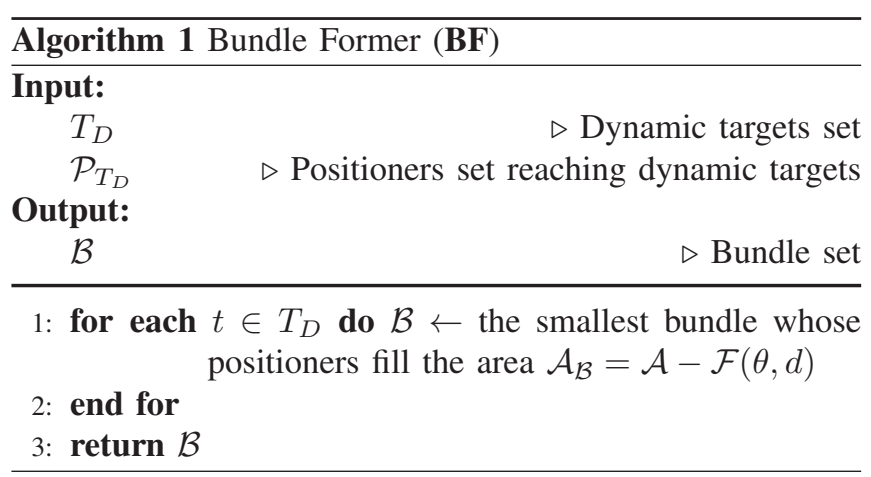

elements which fulfills (4). As an example, we determine the function $\mathcal{F}(\cdot, \cdot)$ for a particular circular bundle geometry. We would like to find the radius of the bundle such that the surface area visible from the bundle's field of view will be $\mathcal{A}$. Note that $\mathcal{A}=\pi(r+x)^{2}$ where $x=d \tan \theta$. Thus, we have

$$
\begin{aligned}
\mathcal{A} & =\pi(r+d \tan \theta)^{2}, \\
& =\pi r^{2}+\pi d \tan \theta(2+d \tan \theta),
\end{aligned}
$$

which turns out that $\mathcal{F}(\theta, d)=\pi d \tan \theta(2+d \tan \theta)$.

We establish the bundle former (BF) algorithm as depicted by Algorithm 1. To analyze the computational complexity of the BF algorithm, we first introduce the notion of "reachability relation" as below.

Definition 5 [Reachability Relation]. Let $t \in T$ be a target. Given a set of positioners $\mathcal{P}$, let $\pi \in \mathcal{P}$ be a positioner. Then, the binary reachability relation $\mathcal{R}(t, \pi)$ holds if $t$ is reachable by $\pi$.

We use the definition above to define the notion of "reachable target set" as follows.

Definition 6 [Reachable Target Set]. Let $T$ be an arbitrary set of targets. Let also $\mathcal{P}$ be a set of positioners. Then, the reachable target set $\mathcal{P}_{T}$ with respect to $T$ is defined as follows.

$$
\mathcal{P}_{T}:=\{\pi \in \mathcal{P} \mid(\forall t \in T) \mathcal{R}(t, \pi)\}
$$

Lemma 1 also proves that the computational complexity of the $\mathbf{B F}$ algorithm is multilinear.

Lemma 1. Let $T_{D}$ and $\mathcal{P}_{T_{D}}$ be a set of dynamic targets and the set of positioners which reach them, respectively. Given $\left|T_{D}\right|:=n$ and $\left|\mathcal{P}_{T_{D}}\right|:=m$, the computational complexity of the $\boldsymbol{B F}$ algorithm is $\mathcal{O}(m n)$.

Proof. Each positioner can only be assigned to one dynamic target. Then in the worst case, each positioner has to be checked to be assigned to every dynamic target. Then, the number of applied tests is $n m$. Therefore, the computational complexity is $\mathcal{O}(m n)$.

\section{B. Algorithm}

In this section, we use the $\mathbf{B F}$ algorithm to propose our heterogeneous assignment algorithm. For this purpose, we

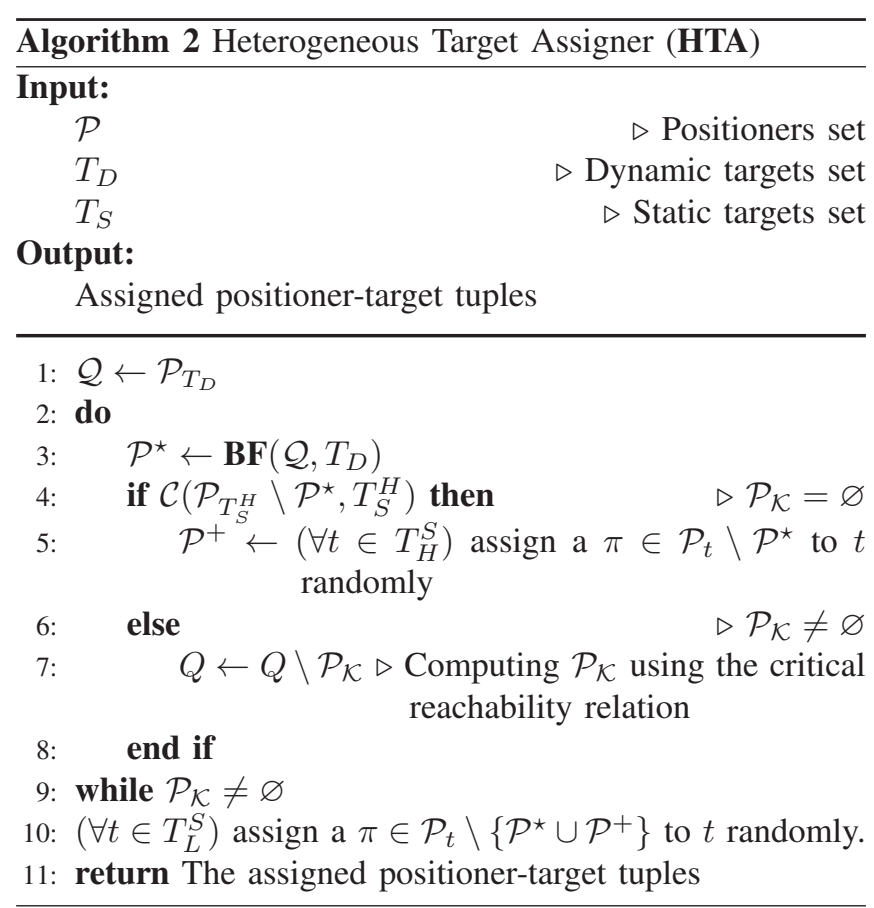

first complete the set of required definitions by introducing "complete reachability" and "critical reachability" relations.

Definition 7 [Complete Reachability]. Let $\mathcal{P}$ and $T$ be a set of positioners and a set of targets, respectively. Then, the complete reachability relation $\mathcal{C}(\mathcal{P}, T)$ holds if the following formula is true.

$$
(\forall t \in T)(\exists \pi \in \mathcal{P}) \mathcal{R}(t, \pi)
$$

Definition 8 [Critical Reachability]. Let $\pi \in \mathcal{P}$ be a positioner. Let also $t^{\prime} \in T_{D}$ and $t^{\prime \prime} \in T_{S}^{H}$ be a dynamic and a high-priority static target, respectively. Then, $\pi$ is critically reachable, say, the ternary relation $\mathcal{K}\left(\pi, t^{\prime}, t^{\prime \prime}\right)$ holds, if the following formula is true.

$$
\left[\pi \in\left(\mathcal{P}_{t^{\prime}} \dot{\cup} \mathcal{P}_{t^{\prime \prime}}\right)\right] \cap\left[\left|\mathcal{P}_{t^{\prime}}\right|=1\right] \cap\left[\left|\mathcal{P}_{t^{\prime \prime}}\right|=1\right]
$$

Definition 9 [Critical Positioner]. Let $\mathcal{P}$ be a set of positioners. Let also $T_{D}$ and $T_{S}^{H}$ be a set of dynamic and a set of high-priority static target, respectively. Then, critical positioner set of $\mathcal{P}$ with respect to $T_{D}$ and $T_{S}^{H}$ is defined as below.

$$
\mathcal{P}_{\mathcal{K}}:=\left\{\pi \in \mathcal{P} \mid\left(\forall t^{\prime} \in T_{D}\right)\left(\forall t^{\prime \prime} \in T_{S}^{H}\right) \mathcal{K}\left(\pi, t^{\prime}, t^{\prime \prime}\right)\right\}
$$

Remark 2. A critical positioner is the one which is the exclusive option to reach a pair of dynamic and high-priority static targets. Since we are interested in the assignment of high-priority static targets ahead of dynamic targets, our heterogeneous assignment algorithm is authorized to assign a positioner to a dynamic target only if that positioner is not critical.

We present our heterogeneous target assignment (HTA) algorithm as depicted in Algorithm 2.

The algorithm in fact generates bundles of the largest possible set of positioners which reach its desired dynamic 
targets (Line 3). If the remaining positioners fulfill the complete reachability requirements of all high-priority static targets (Line 4), the algorithm assigns them to those targets properly (Line 5). Otherwise, if the complete reachability is not achieved (Line 6), one implies that some of the assigned positioners to dynamic targets are critical. So, the algorithm excludes those critical positioners by assessing the critical reachability relation (Line 7 ). Then, the bundles are recomputed with a new restricted set of reachable positioners which no longer includes any critical positioner. One notes that the next attempt shall be successful to assign at least one positioner to each of the high-priority static targets. In end end, the remainder of unassigned positioners may be assigned to some low-priority static targets (Line 10).

Now, we show that the computational complexity of the HTA algorithm is multilinear.

Proposition 2. Let $\mathcal{P}, T_{D}$, and $T_{S}$ be a set of positioners, a set of dynamic targets, and a set of static targets, respectively. Given $m:=|\mathcal{P}|, n:=\left|T_{D}\right|$, and $k:=\left|T_{S}\right|$, the computational complexity of the HTA algorithm is $\mathcal{O}(m n k)$.

Proof. To take the worst case into account, we assume the $T_{S}^{L}=\varnothing$ which means $T_{S}=T_{S}^{H}$ implying that all of the static targets are high-priority in view of the assignment process. An upper bound for the cardinality of $\mathcal{Q}$ is $m=|\mathcal{P}|$. Additionally, according to the result of Lemma 1, bundle formation requires $m n$ units of time. Checking the completeness condition requires the iteration over both $T_{D}$ and $T_{S}$ sets, thereby $m n$ units of time shall be spent. Thus, the assignment process associated with high-priority static targets shall be done during $m n k$ units of time. The loop of the algorithm is executed one and two times in the best and in the worst cases, respectively. Since we assumed that all static targets are high-priority, the loop is executed twice, and there is no low-priority static target to which a positioner is assigned. Therefore, the overall worst case computational complexity of the HTA algorithm is

$$
\mathcal{O}(2(m n+m n k)) \equiv \mathcal{O}(m n k) .
$$

\section{Simulation}

In this section, we solve a heterogeneous target assignment problem using the HTA algorithm. In particular, we assume a focal plane including 91 robotic fiber positioners. We also assume 2 dynamic targets which are two hypothetical cubesat satellites [13] operating for the purpose of active space debris removal at the range of the LEO orbit. We take overall 20 static targets, say, galaxies to be observed, 12 of which are high-priority, and the others are low-priority. A normal distribution is used to generate the relative locations of the static targets with respect to the focal plane. We assume the robotic positioners of the SDSS-V project [6] the length of each of which is $350 \mathrm{~mm}$. Furthermore, the vertical distance between the ferrule and the first axis of each positioner is 15

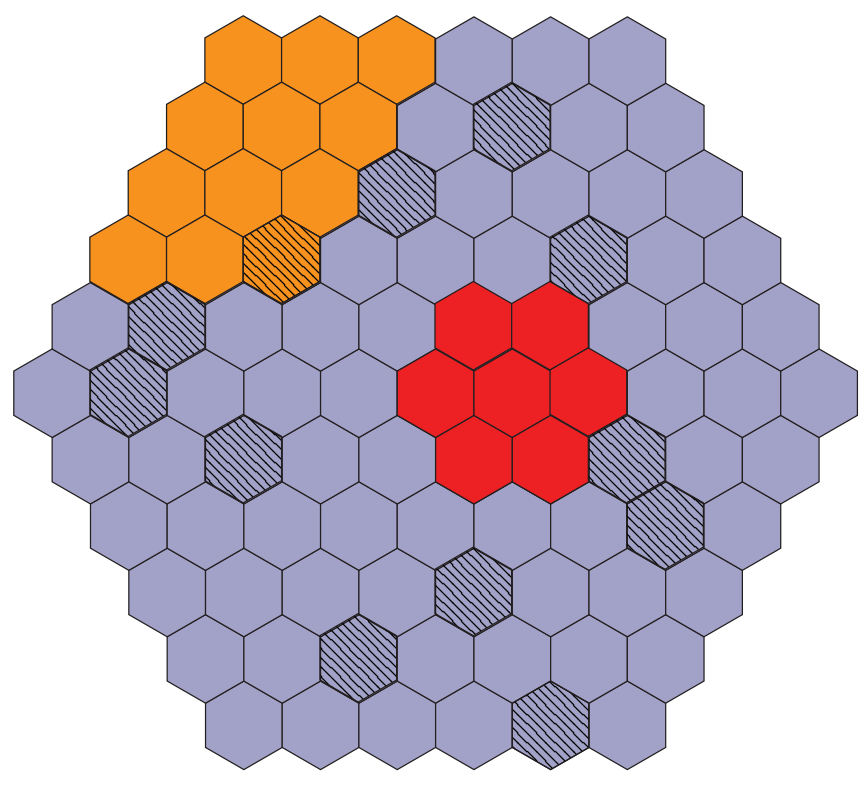

Fig. 4. The assignment results after the first iteration of the algorithm's loop (The red and yellow positioners correspond to the bundles of the first and the second dynamic targets, respectively. Th crossed positioners are those which must be assigned to high-priority static targets.)

$\mathrm{mm}$. Then considering the tilt rate of $\sim 0.35^{\circ}$, the overall deviation of a positioner's ferrule is $\sim 0.05 \mathrm{rad}$. We assume that all of the positioners are identical, and the distance $d$ associated with the first and the second dynamic targets are $1200 \mathrm{~km}$ and $500 \mathrm{~km}$, respectively. The radii of the surfaces around the first and the second dynamic target are required to be $48 \mathrm{~km}$ and $12 \mathrm{~km}$, respectively.

The evolution of the solution to the problem is as follows. The first iteration of the algorithm's loop assigns a bundle to each dynamic target. The bundles are depicted in Figure 4. In particular, the red bundle and the orange bundle correspond to the first and the second dynamic targets, respectively. However, the crossed positioners are those which have to be assigned to high-priority static targets. As the figure illustrates, one of the orange positioners is a critical one. Thus, the second iteration of the loop removes that positioner from the valid options to shape a bundle, thereby assigning it to the desired high-priority static target. Note that this removal decreases the area of the surface corresponding to the second dynamic target, but it is unavoidable since the critical positioner has to be assigned to the high-priority static target. The second iteration of the algorithm is depicted in Figure 5. In this figure, the double crossed positioners are those which may be assigned to low-priority static targets. However, one observes that this potential assignment required two positioners which has been already assigned to dynamic targets. Thus, the algorithm neglects those two low-priority static targets, and the final result is illustrated in Figure 6.

\section{Conclusions}

Robotic fiber positioners have already been used to generate the map of the observable universe. Space applications, 


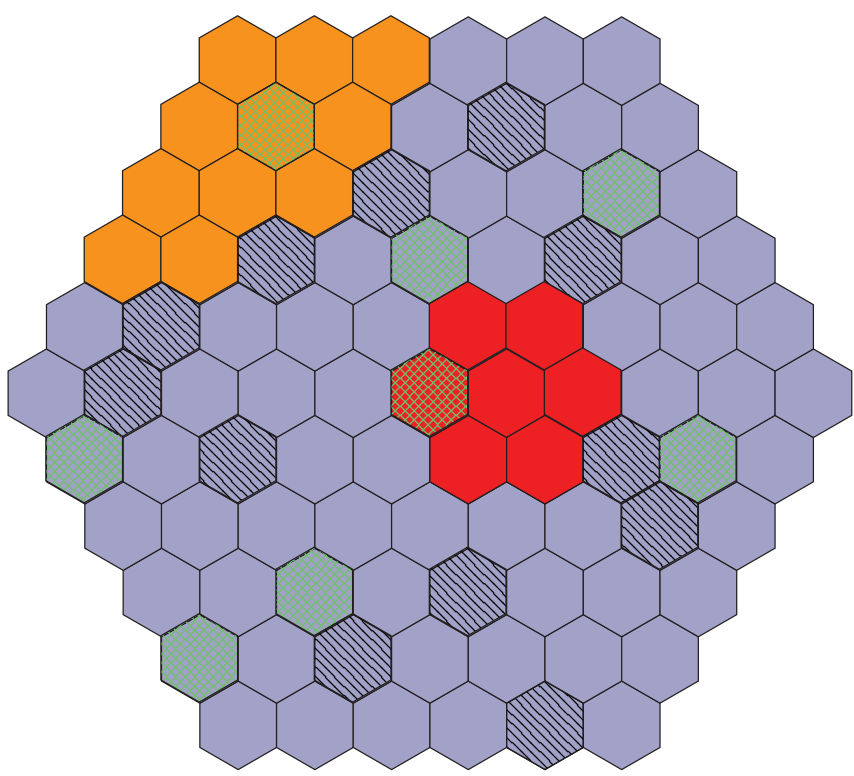

Fig. 5. The assignment results after the second iteration of the algorithm's loop (The red and yellow positioners correspond to the bundles of the first and the second dynamic targets, respectively. The crossed and the double crossed positioners are those which must be assigned to high-priority and low-priority static targets, respectively.)

such as debris detection, can also benefit from these robotic fiber positioners. This research presented an algorithm which assigns the robotic fiber positioners of a telescope to various types of targets, from observational to space operational ones. Thus, not only a wider range of applications can be handled by robotic fiber positioners but the overall information throughout of a typical positioners set is maximized since more robotic fiber positioners are used at the same time.

One may note that robotic fiber positioners may need various exposure times to collect signals from different types of targets depending on their nature. As a future work, the algorithm may be upgraded such that the distance between positioners batches corresponding to those heterogeneous targets whose exposure times are close to each other will be maximized. Thus, a typical spectrograph can efficiently distinguish between the traces of the objects corresponding to each of the intended heterogeneous applications.

\section{REFERENCES}

[1] E. Mazets, S. Golenetskii, V. Ilyinskii, Y. A. Guryan, R. Aptekar, V. Panov, I. Sokolov, Z. Y. Sokolova, and T. Kharitonova, "Cosmic gamma-ray burst spectroscopy," Astrophysics and Space Science, vol. 82, no. 2, pp. 261-282, 1982.

[2] A. Aghamousa, J. Aguilar, S. Ahlen, S. Alam, L. E. Allen, C. A. Prieto, J. Annis, S. Bailey, C. Balland, O. Ballester, et al., "The desi experiment part i: Science, targeting, and survey design," arXiv preprint arXiv:1611.00036, 2016.

[3] A. Aghamousa, J. Aguilar, S. Ahlen, S. Alam, L. E. Allen, C. A. Prieto, J. Annis, S. Bailey, C. Balland, O. Ballester, et al., "The desi experiment part ii: Instrument design," arXiv preprint arXiv:1611.00037, 2016.

[4] M. Cirasuolo, J. Afonso, M. Carollo, H. Flores, R. Maiolino, E. Oliva, S. Paltani, L. Vanzi, C. Evans, M. Abreu, et al., "Moons: the multiobject optical and near-infrared spectrograph for the vlt," in Groundbased and airborne instrumentation for astronomy $V$, vol. 9147, p. $91470 \mathrm{~N}$, International Society for Optics and Photonics, 2014.

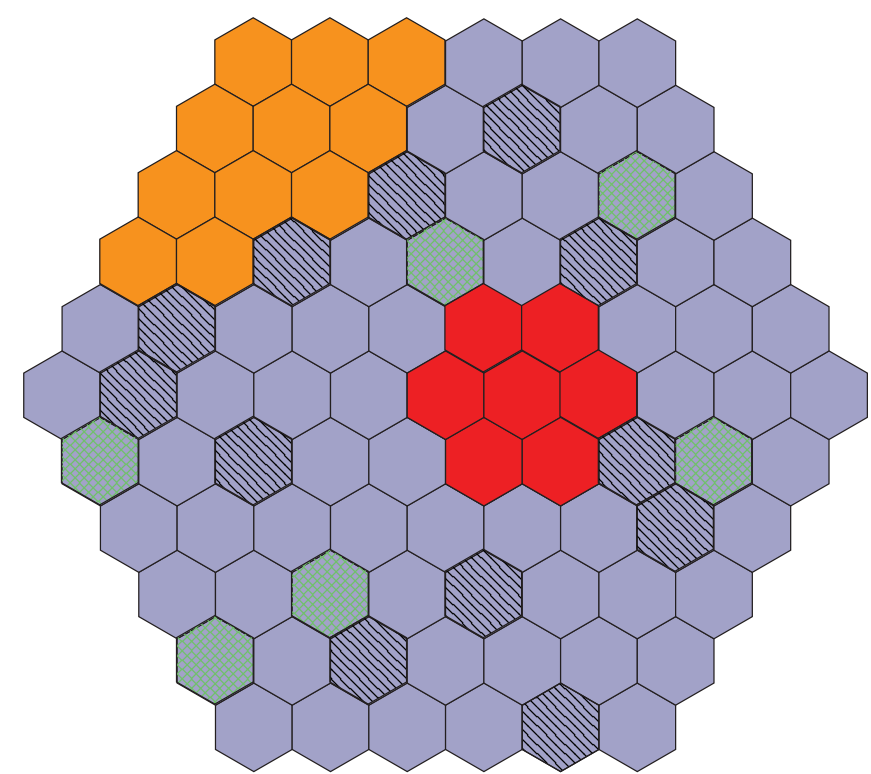

Fig. 6. The final results of the assignment process (The red and yellow positioners correspond to the bundles of the first and the second dynamic targets, respectively. The crossed and the double crossed positioners are those which must be assigned to high-priority and low-priority static targets, respectively.)

[5] M. Takada, R. S. Ellis, M. Chiba, J. E. Greene, H. Aihara, N. Arimoto, K. Bundy, J. Cohen, O. Doré, G. Graves, et al., "Extragalactic science, cosmology, and galactic archaeology with the subaru prime focus spectrograph," Publications of the Astronomical Society of Japan, vol. 66 , no. $1,2014$.

[6] M. Macktoobian, D. Gillet, and J.-P. Kneib, "The navigation of robotic fiber positioners in sdss-v project: Design and implementation," in 2019 15th Conference on Ph. D Research in Microelectronics and Electronics (PRIME), pp. 85-88, IEEE, 2019.

[7] M. Macktoobian, D. Gillet, and J.-P. Kneib, "Supervisory coordination of robotic fiber positioners in multi-object spectrographs," in The Proceedings of the the 15th IFAC Symposium on Large Scale Systems (LSS): Theory and Applications, no. CONF, Elsevier, 2019.

[8] P. Hörler, L. Kronig, J.-P. Kneib, M. Bouri, H. Bleuler, and D. von Moos, "High density fiber postitioner system for massive spectroscopic surveys," Monthly Notices of the Royal Astronomical Society, vol. 481, no. 3, pp. 3070-3082, 2018.

[9] S. Doeleman, E. Agol, D. Backer, et al., "Astro2010: The astronomy and astrophysics decadal survey," Science White Papers, vol. 68, 2009.

[10] I. Morales, A. D. Montero-Dorta, M. Azzaro, F. Prada, J. Sánchez, and $\mathrm{S}$. Becerril, "Fibre assignment in next-generation wide-field spectrographs," Monthly Notices of the Royal Astronomical Society, vol. 419, no. 2, pp. 1187-1196, 2011.

[11] C. E. Schaefer, L. Makarem, and J.-P. Kneib, "Target-based fiber assignment for large survey spectrographs," in Software and Cyberinfrastructure for Astronomy IV, vol. 9913, p. 991335, International Society for Optics and Photonics, 2016.

[12] D. Tao, L. Makarem, M. Bouri, J.-P. Kneib, and D. Gillet, "Priority coordination of fiber positioners in multi-objects spectrographs," in Ground-based and Airborne Instrumentation for Astronomy VII, vol. 10702, p. 107028K, International Society for Optics and Photonics, 2018.

[13] C. Pirat, M. Richard-Noca, C. Paccolat, F. Belloni, R. Wiesendanger, D. Courtney, R. Walker, and V. Gass, "Mission design and gnc for in-orbit demonstration of active debris removal technologies with cubesats," Acta Astronautica, vol. 130, pp. 114-127, 2017.

[14] M. Kimura, T. Maihara, F. Iwamuro, M. Akiyama, N. Tamura, G. B. Dalton, N. Takato, P. Tait, K. Ohta, S. Eto, et al., "Fibre multi-object spectrograph (fmos) for the subaru telescope," Publications of the Astronomical Society of Japan, vol. 62, no. 5, pp. 1135-1147, 2010. 